\title{
A case of epididymal sarcoidosis
}

\author{
Lisa G. Smyth, MD; Ronan M. Long, MD; Gerald Lennon, MD
}

Department of Urology, St.Vincent's University Hospital, Dublin, Ireland

Cite as: Can Urol Assoc J 2011;5(5):E90-E91; D01:10.5489/cuaj.10126

\begin{abstract}
Sarcoidosis is a systemic inflammatory condition that primarily affects the lungs, lymphatic system and skin. Extra thoracic manifestations occur in about $50 \%$ of cases; however, it rarely affects the male reproductive tract. The first documented case of epididymal and testicular sarcoid in the Irish literature is of a 25-year-old male who presented with a painful $1-\mathrm{cm}$ extra-testicular scrotal mass. While the involvement of the male reproductive tract is rare, it should be included in the differential of testicular pathology. It can be diagnosed on frozen section and may be managed conservatively.
\end{abstract}

\section{Introduction}

Sarcoid is a multisystem disease of unknown cause characterized by non-caseating granulomas. It affects the genitourinary system in $0.2 \%$ of cases; the kidney is the most common genitourinary organ affected. Testicular sarcoid is rare. We report the case of a 25-year-old whose first presentation with sarcoid was an epididymal mass.

\section{Case presentation}

A 25-year-old male presented with a painful scrotal mass. On examination, he had a firm 1-cm solid right epididymal nodule and no lymphadenopathy. A scrotal ultrasound revealed a 1-cm solid lesion in the head of the right epididymis. His serum tumour markers were normal. He developed abdominal pain 3 weeks later and a computed tomography (CT) of the KUB (kidney, ureter, bladder) demonstrated bibasal ill-defined lung opacities. A CT thorax, abdomen and pelvis revealed hilar and mediastinal lymphadenopathy, but no retroperitoneal lymphadenopathy. A mediastinoscopy and biopsy established sarcoid. A repeat scrotal ultrasound 3 months later demonstrated expansion of the lesion $(2.8 \times 1.7 \times 1.7 \mathrm{~cm})$ from the head to the epididymal body invaginating into the right testicle.
He underwent a scrotal exploration (inguinal approach) and frozen section to out-rule malignancy. This confirmed epididymal and testicular sarcoidosis. His serum angiotensin converting enzyme (ACE) was elevated at $86 \mathrm{U} / \mathrm{L}$ (normal range 15-70 U/L) and he was referred to a respiratory physician. He is currently well and has not been started on steroids.

\section{Discussion}

Sarcoid is a systemic inflammatory disorder characterized by non-caseating granulomas most commonly found in the lung and lymphatic system. It is thought to occur as a result of chronic immunological response to infectious or environmental stress; there may also be a genetic association. ${ }^{1}$

Extra-thoracic sarcoid occurs in about $50 \%$ of cases. The most frequent sites affected are peripheral lymph nodes, skin, eyes and liver. There are many unusual sites documented in the literature, including tattoos, breast, scars and joints. ${ }^{2-5}$ Sarcoid involving the male reproductive tract is rare. The first case of testicular sarcoid was described in $1936 .{ }^{6}$ Over $50 \%$ of genitourinary sarcoid is found in African-American men with less than $10 \%$ of cases in Caucasian men, ${ }^{7}$ therefore our case is unusual as the patient was Caucasian.

A review of 60 cases by Kodama and colleagues revealed that the average age at onset is 33 years; it is more common in African-American men (58\%), with $73 \%$ of cases involving the epididymis and $43 \%$ the testes. In this study, 21 men underwent unnecessary orchidectomy or epididymectomy; ${ }^{7}$ however, we managed our patient conservatively.

A retrospective study of 1120 patients with germ cell tumours found 9 patients had sarcoid in the presence of a testicular tumour. There is evidence that sarcoid increases the risk of lung cancer and lymphoma; ${ }^{8}$ however, there is no causative link with testicular cancer. A study at the Mayo Clinic demonstrated a 100-fold increased risk of developing sarcoid after treatment for testicular cancer. ${ }^{9}$ However, the regular radiological examination increases the likelihood of 


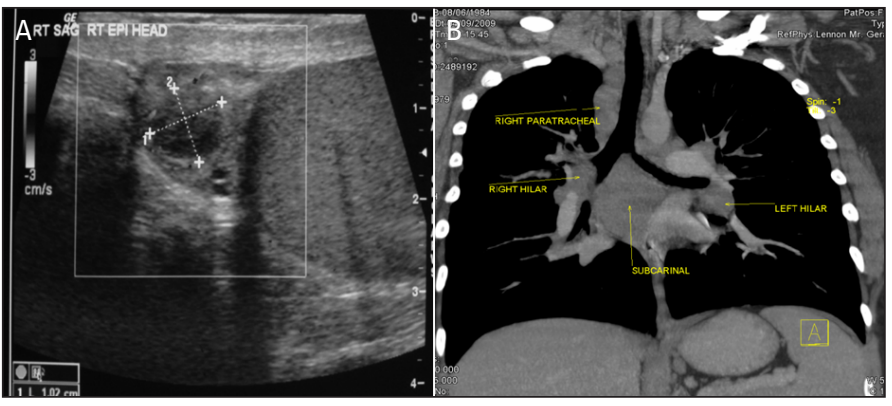

Fig. 1. A: Scrotal ultrasound demonstrating a $1 \mathrm{~cm}$ hypoechoic lesion in the right epididymal head. B: CT thorax revealed hilar and mediastinal lymphadenopathy.

detection and this must be considered in the interpretation of the 100-fold increased risk.

Sarcoid diagnosed after treatment of testicular carcinoma may be confused with metastases. Positron emission tomography may incorrectly pick up sarcoid inflammation rather than testicular metastases. ${ }^{10}$ It is important to take multiple biopsies to avoid inappropriate treatment with chemotherapy. ${ }^{11}$ The risks associated with biopsy are less than those with unnecessary chemotherapy.

Sarcoid should be included in the differential of a scrotal mass. Suspicion of sarcoid should also be raised if the patient is African-American, has normal tumour markers, a raised serum ACE or involvement of the epididymis. Where sarcoid is suspected, a biopsy and frozen section should be sent prior to orchidectomy.

\section{Conclusion}

Sarcoid may affect the male reproductive tract, presenting most commonly as an epididymal or testicular mass. While testicular cancer must always be ruled out, sarcoid should be included in the differential diagnosis.

This case demonstrates the need for histological confirmation prior to orchidectomy, particularly when the epididymal is involved; in contrast to testicular cancer, testicular sarcoid may be managed conservatively to preserve fertility.

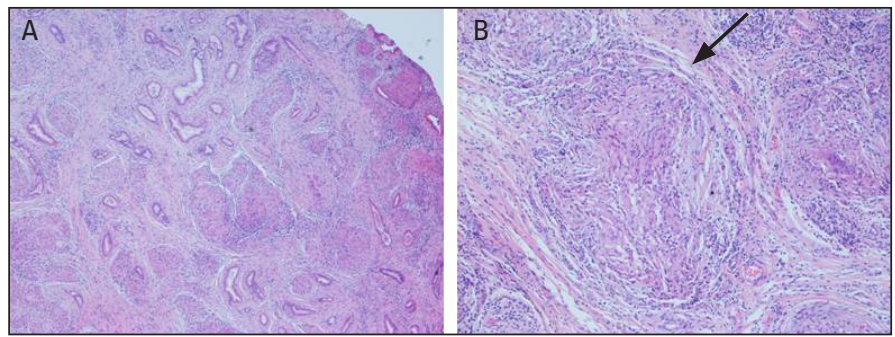

Fig. 2. High (A) and low (B) magnification of testicular sarcoid with the characteristic non-caseating granulomas (arrow).

Competing interests: None declared.

This paper has been peer-reviewed.

\section{References}

1. Priestley S, Delaney JC. Familial sarcoidosis presenting with stridor. Thorax 1981;36:636-7.

2. Ali S, Gilliam AC, Brodell RT. Sarcoidosis appearing in a tattoo. I Cutan Med Surg 2008;12:43-8.

3. Fiorucci F, Conti V, Lucantoni $G$, et al. Sarcoidosis of the breast: a rare case report and a review. Eur Rev Med Pharmacol 2006; 10:47-50.

4. Singal A, Thami GP, Goraya IS. Scar sarcoidosis in childhood: case report and review of the literature. Clin Exp Dermatol 2005;30:244-6.

5. Veale D, Fitzgerald 0. Acute sarcoid arthropathy-an infective cause? Br J Rheumatol 1990;29:158-9.

6. Schaumann J. Lymphogranulomatosis benigna in the light of prolonged clinical observations and autopsy findings. Br J Dermatol 1936;48:399-446.

7. Kodama K, Hasegawa $\mathrm{T}$, Egawa M, et al. Bilateral epididymal sarcoidosis presenting without radiographic evidence of intrathoracic lesion: Review of sarcoidosis involving the male reproductive tract. Int I Urol 2004; 11:345-8.

8. Brinker H. Coexistence of sarcoidosis and malignant disease: Causality or coincidence? Sarcoidosis 1989;6:31-43

9. Rayson D, Burch PA, Richardson RL. Sarcoidosis and testicular carcinoma. Cancer 1998;83:337-43.

10. Karapetis C, Strickland AH, Yip D, et al. PET and PLAP in suspected testicular cancer relapse: beware sarcoidosis. Ann Oncol 2001;12:1485-8.

11. Tjan-Heijnen V, Vlasveld LT, Pernet FP, et al. Coincidence of seminoma and sarcoidosis: a myth or fact? Ann Oncol 1998;9:321-5.

Correspondence: Dr. Lisa G. Smyth, Limerick Regional Hospital, Dooradoyle, Co Limerick, Ireland; elizabethsmyth@rcsi.ie 